\title{
A Concurrent Region Growing Algorithm Guided by Circumscribed Contours
}

\author{
X.Cufí, X.Muñoz, J.Freixenet and J.Martí \\ Computer Vision and Robotics Group. liiA - EIA. University of Girona \\ $\{x c u f, x m u n o z, j o r d i f$, joanm $\} @ e i a . u d g . e s$
}

\begin{abstract}
Image segmentation of natural scenes constitutes a major problem in Machine Vision. This paper presents a new proposal for the image segmentation problem which has been based on the integration of edge and region information. This approach begins by detecting the main contours of the scene which are later used to guide a concurrent set of growing processes. A previous analysis of the seed pixels permits adjustment of the homogeneity criterion to the region's characteristics during the growing process. Since the high variability of regions representing outdoor scenes makes the classical homogeneity criteria useless, a new homogeneity criterion based on clustering analysis and convex hull construction is proposed. Experimental results have proven the reliability of the proposed approach.
\end{abstract}

\section{Introduction}

During the last few years many image segmentation techniques focusing on region-based and edge-based segmentation have been developed [1]. Unfortunately, these techniques still fail to produce an accurate segmentation. Region-based techniques often fail to yield the desired structure due to the difficulty of choosing reasonable starting "seed" points and appropriate growing rules. Similarly, edge-based techniques often fail as a consequence of object similarity within the scene or indistinct boundaries among image objects.

As a result, current trends in segmentation try to integrate several techniques in order to produce better results. Two different ways of integration are analysed in this paper. The first consists of refining the obtained segmentation using the results of another segmentation technique. The edge information, for example, can be used to refine a region segmentation process [2], or alternatively a region-based segmentation can be used to automatically add edge segments into a fully connected line-drawing [3]. The second way is based on integrating edge detection and region extraction in the same process [4].

Our proposal follows the second approach using the edges of the image as a guide to orientate the later region growing process properly. Suppose a contour separates two regions. A set of germs is then located at both sides of the contour in order to locate some features of the region. This prior knowledge allows the adjustment of the region growing algorithm and the resolution of a very common problem: the difficulty of establishing an adequate criterion of homogeneity. Classical algorithms base their decision of merging a region with a pixel on a homogeneity criterion, which usually takes into consideration some statistical parameters on the regions. However, this criterion often may not be applicable to highly textured images or range images [5] because regions in such images usually have very different homogeneity behaviours, as is depicted in Figure 1.
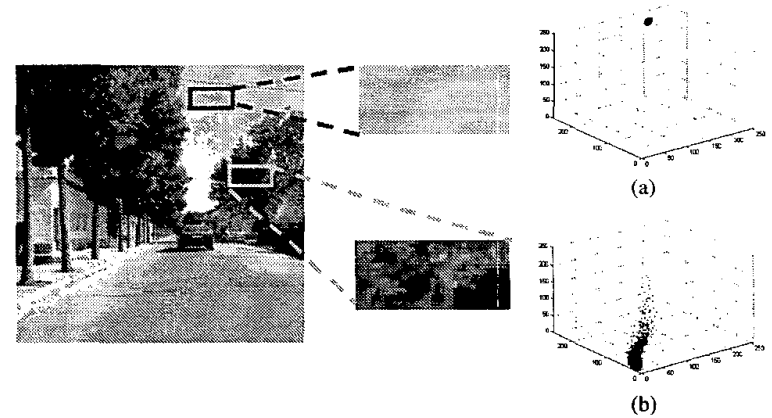

Figure 1. Two chromatic feature spaces corresponding to the imaged objects sky (a) and leaves (b), depicting different homogeneity behaviour.

Therefore, looking for a single criterion becomes an inadequate procedure. Our approach allows individual adjustment of the criterion to the region and the use of different criterion in each case.

\section{Overview}

The scheme of the technique proposed consists of four basic steps (see Figure 2). The first concentrates on main contour extraction and focuses on detecting the boundaries between the different regions of the image and filtering the edges with less relevance. The result is the extraction of the most relevant contours in the image. 
Getting these contours is a really difficult job but it is essential for further processing.

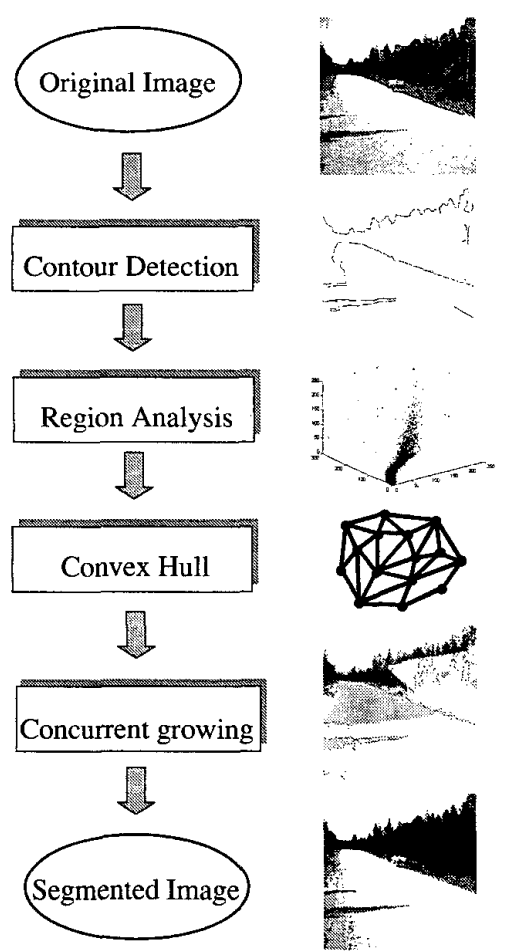

Figure 2. Scheme with the basic steps of the proposed technique.

At the beginning of the second step, a number of seeds are launched inside the supposed region and used as samples. The seeds are then analysed using a hierarchical clustering algorithm with the objective of obtaining the clusters they generate in the chromatic space. This knowledge is further used in the third stage to construct the convex hull of each cluster. The convex hull establishes the shape of each cluster and is the base for the homogeneity criterion. The algorithm bases its decision to merge a pixel with the region on the relative placement of the pixel (inside-outside) in respect to the convex hulls associated with the samples of the region. Finally, in the fourth step, the seeds simultaneously start a concurrent growth using the criterion established for each region.

\section{Contour Detection}

The goal of the contour detection step is to obtain a set of boundaries of the most significant regions perceived, named the Circumscribed Contours of the image. The contour detection and extraction is realised according to the algorithm proposed in [6]. The method is based on the two most relevant properties presented by these
Circumscribed Contours: First, principal contours must have an outstanding length within the global frame of the image. Secondly, the regions separated by the contours should present some appreciable differences in chromatic and textural features.

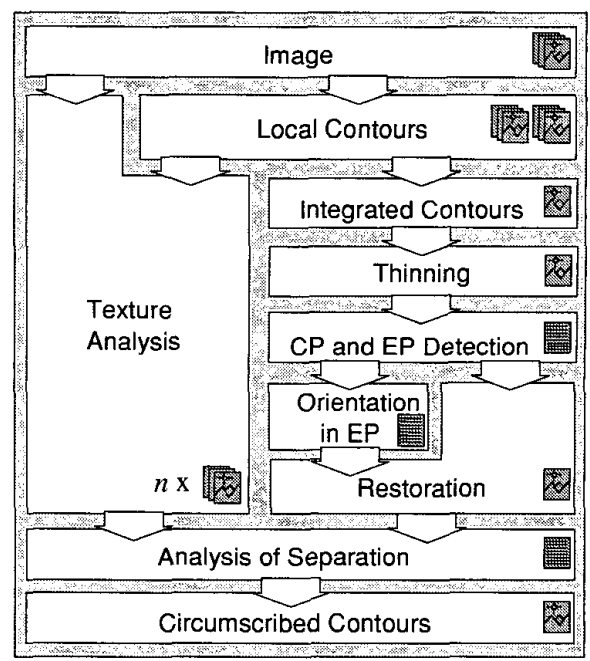

Figure 3. Scheme of process obtaining Circumscribed Contours. $\mathrm{CP}=$ Cross points, $\mathrm{EP}=$ End points.

The process followed to obtain the Circumscribed Contours is shown in Figure 3. The basic phases of processing are: 1) Obtaining the Integrated Contour Image, 2) Restoration of the contours, 3) Texture Analysis, and 4) Analysis of possible separation of regions.

\section{Region Analysis}

The contours obtained in the previous step represent the boundaries between regions. Every contour theoretically separates two adjacent regions in the image. The growing centres (called seeds) are chosen at each side and along the contour, as is illustrated in Figure 4a. Here, we suppose the seeds belonging to a determined side of the contour are associated to the same region. Then, these seeds are used as samples of the corresponding regions and analysed in the chromatic space. Figure $4 \mathrm{~b}$ shows a set of seed points, which represent the characteristics of the region located at one side of the contour, mapped into the RGB feature space.

One essential characteristic of the distribution is the number of clusters which fit the samples. Each cluster has its own identity and must be considered individually. The region analysis tries to determinate the clustering in the region. A classic clustering algorithm is used with this objective. Hierarchical clustering constructs a hierarchical 
structure by iteratively merging clusters according to certain dissimilarity measurements (concretely the Euclidean distance), starting from singleton elements until no further merging is possible.

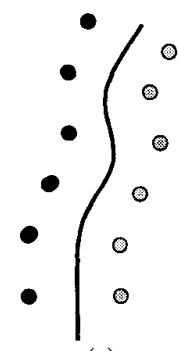

(a)

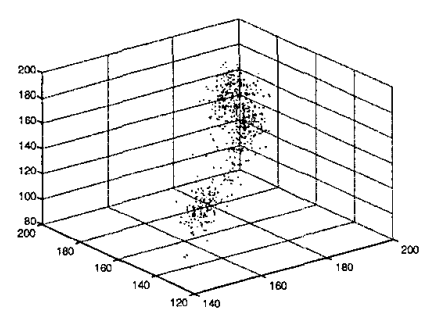

(b)
Figure 4. Two sets of seeds located at both sides of a contour (a), and the representation of one set in the chromatic space RGB in (b).

The identification of the number of clusters is realised using the sequence of clustering generated. An intuitive approach is to search for clusters which have a long lifetime. The lifetime of a cluster is defined as the absolute value of the difference between the proximity level which has been created and the proximity level when it is absorbed into a large cluster. The analysis of the seeds gives us the number of clusters and the seeds which are grouped in each cluster. This information is essential for the next step, where the most convenient criterion of homogeneity for the region, is fixed.

\section{The criterion of homogeneity: Convex Hull}

The convex hull is a well-known concept in computational geometry. By definition, the convex hull of a set of points $S$ is the smallest convex set containing $S$. The convex hull of a set of $\mathrm{N}$ points in three-dimensional space can be computed in optimal time $\theta(\mathrm{N} \log N)$ [7]. Each cluster of points is treated individually and a convex hull is obtained for each one. This process defines the shape of each cluster in the chromatic space.

The decision of merging a pixel inside a region is based on the relative placement of the pixel in respect to the convex hulls associated with the region. If the pixel is inside any of the convex hulls the pixel is merged into the region.

\section{Concurrent implementation}

To ensure an efficient and easy implementation, a standard for distributed object oriented computing, CORBA [8], has been chosen. Following this specification, a set of objects-software can be programmed in heterogeneous programming languages, executed at several workstations with different operating systems which cooperate, in order to solve a concrete problem by using a common and simple mechanism.

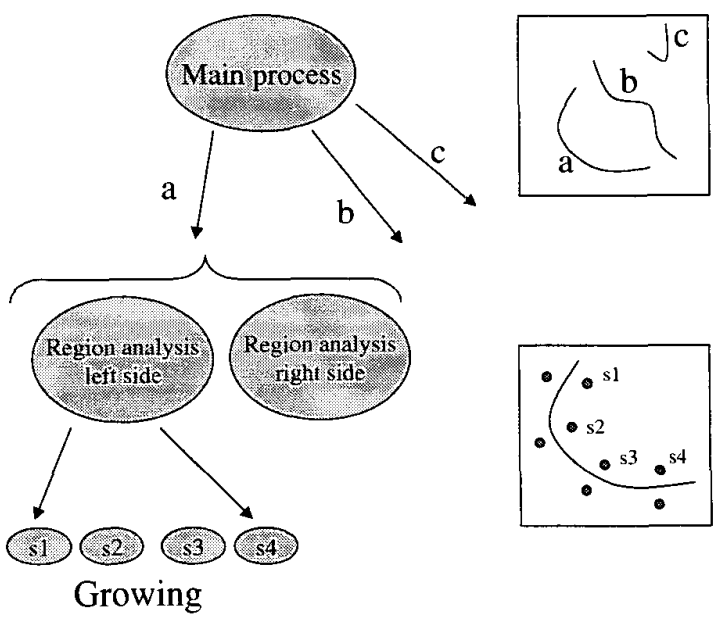

Figure 5. Scheme of the whole set of processes (threads) involved in the algorithm. Each $S_{i}$ is the task engaged to perform a region growing process starting at the pixel seed $i$.

Figure 5 shows a scheme in which the complete set of processes which participate in our proposal appear. Once the main process has detected the whole set of contours, it then creates two threads associated to each contour. These threads are dedicated to performing a specific region analysis at each side of the contour. Then, every thread launches a new set of threads which will perform the region growing process. These last processes, associated with the seeds, compete for the pixels of the image accessing in mutual exclusion. Finally, when all the processes have finished their growing, the algorithm begins to merge neighbouring regions.

\section{Experimental Results}

The proposed segmentation algorithm has successfully segmented a wide range of natural images. Three representative images are shown in this paper: a natural scene with a lake, a semi-structured zone of a road going through a forest, and an urban location.

The results of the proposed segmentation technique (Figure 6b) are compared with the segmentation obtained by means of a classic region growing algorithm using the average as homogeneity criterion (Figure 6a). Note that the proposed technique achieves better results than traditional techniques on textured regions (the forest in the semi-structured image and the lake in the natural image), since the typical algorithm has over-segmented these regions. 

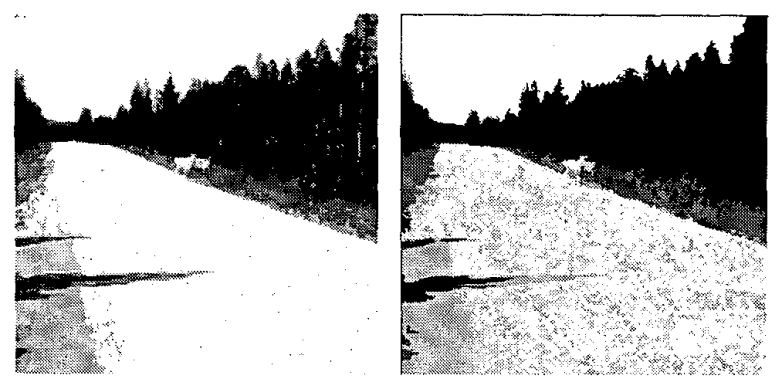

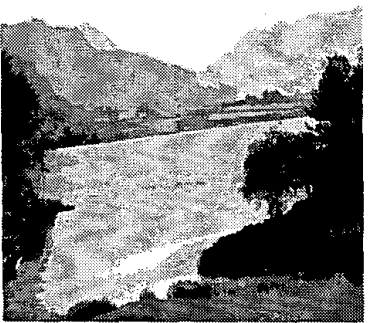

(a)

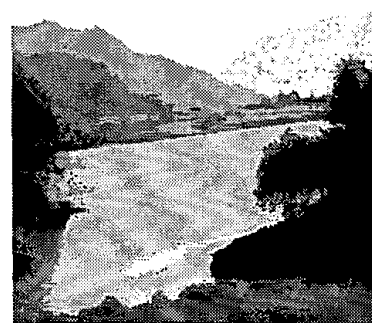

(b)
Figure 6. Comparative segmentation results. The regions obtained by a typical Region Growing in column (a), and the regions obtained by the proposed algorithm (b).

Figure 7 shows the use of a homogeneity criterion adjusted to the specific characteristics of a region which permits grouping the conceptual region inside a single region solving the segmentation of highly textural images.

\section{Conclusions}

In this paper we have presented a method for the segmentation of natural images which integrates region and edge information. We have proposed a new homogeneity criterion for the region growing algorithm based on a previous analysis of the seeds located at the sides of the contours. This criterion is adjusted to the region's characteristics and is based on clustering analysis and construction of convex hulls of the seeds in chromatic space. Experimental results indicate that the proposed method provides much better results than traditional techniques in the segmentation of natural images.

The implementation of this approach has been performed by using the standard CORBA, which will ensure easy evolutions of the system in the near future as well as the necessary current performance for parallel execution of tasks.

\section{References}

[1] R. Haralick, L.Shaapiro, "Survey: Image Segmentation Techniques"; Computer Vision Graphics, Image Processing, vol.29, pp.100-132; 1985.
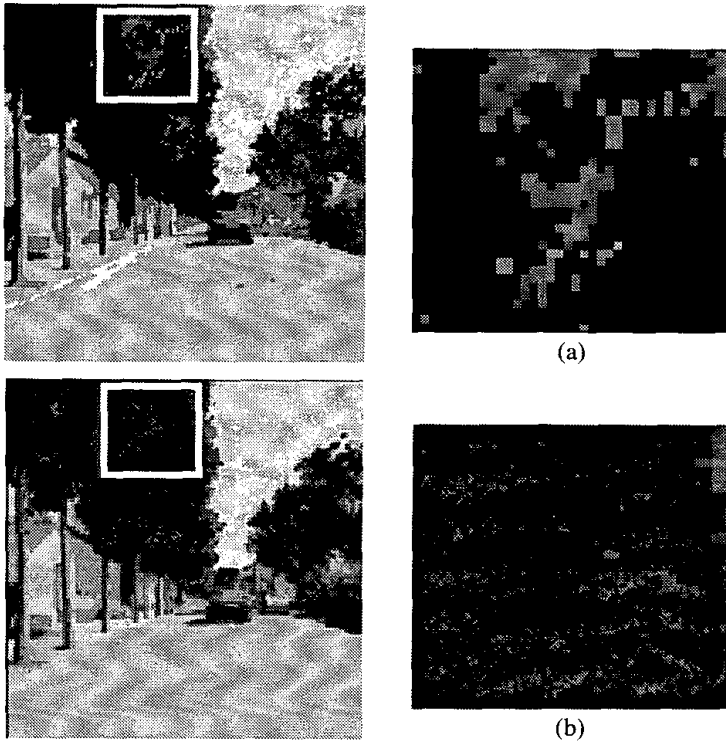

(b)

Figure 7. Textured region segmentation. Segmentation of leaves by a typical homogeneity criterion (a), and the segmentation achieved by the proposed criterion (b)

[2] T. Pavlidis, Y.T. Liow, "Integration Region Growing and Edge Detection"; IEEE Trans. on PAMI 12, No.3, pp 225-233; March 1990.

[3] E.J. Pauwels, P. Fiddelaers, L.J. Van Gool, "Autonomous Grouping of Contour-Segments Using an Adaptive RegionGrowing Algorithm"; Proceedings of ICPR'96, pp.586-590; 1996.

[4] J.P. Gambotto, "A new approach to combining region growing and edge detection; Pattern Recognition Letters 14, pp.869-875; 1993.

[5] R. Adams, L. Bischof, "Seeded Region Growing"; IEEE Trans on PAMI 16, No.6, pp 641-647; June 1994.

[6] X. Cufí, A.Casals, "A Criterion for Circumscribed Contour Determination for Image Segmentation"; $M V A^{\prime} 96$ IAPR Workshop on Machine Vision Applications, pp.309-312; Tokyo, November 1996.

[7] F.P.Preparata and S.J. Hong, "Convex Hulls of Finite Sets of Points in two and three Dimensions"; Comm. ACM 2(20), pp.87-93; February 1977.

[8] S.Baker, CORBA Distributed Objects. Uning Orbix; In ACM Press. Addisson-Wesley; 1997. 